Document downloaded from:

http://hdl.handle.net/10251/47423

This paper must be cited as:

Magin, I.; Polyakov, N.; Khramtsova, E.; Kruppa, A.; Stepanov, A.; Purtov, P.; Leshina, T.... (2011). Spin chemistry investigation of peculiarities of photoinduced electron transfer in donor-acceptor linked system. Applied Magnetic Resonance. 41(2-4):205-220. doi:10.1007/s00723-011-0288-3.

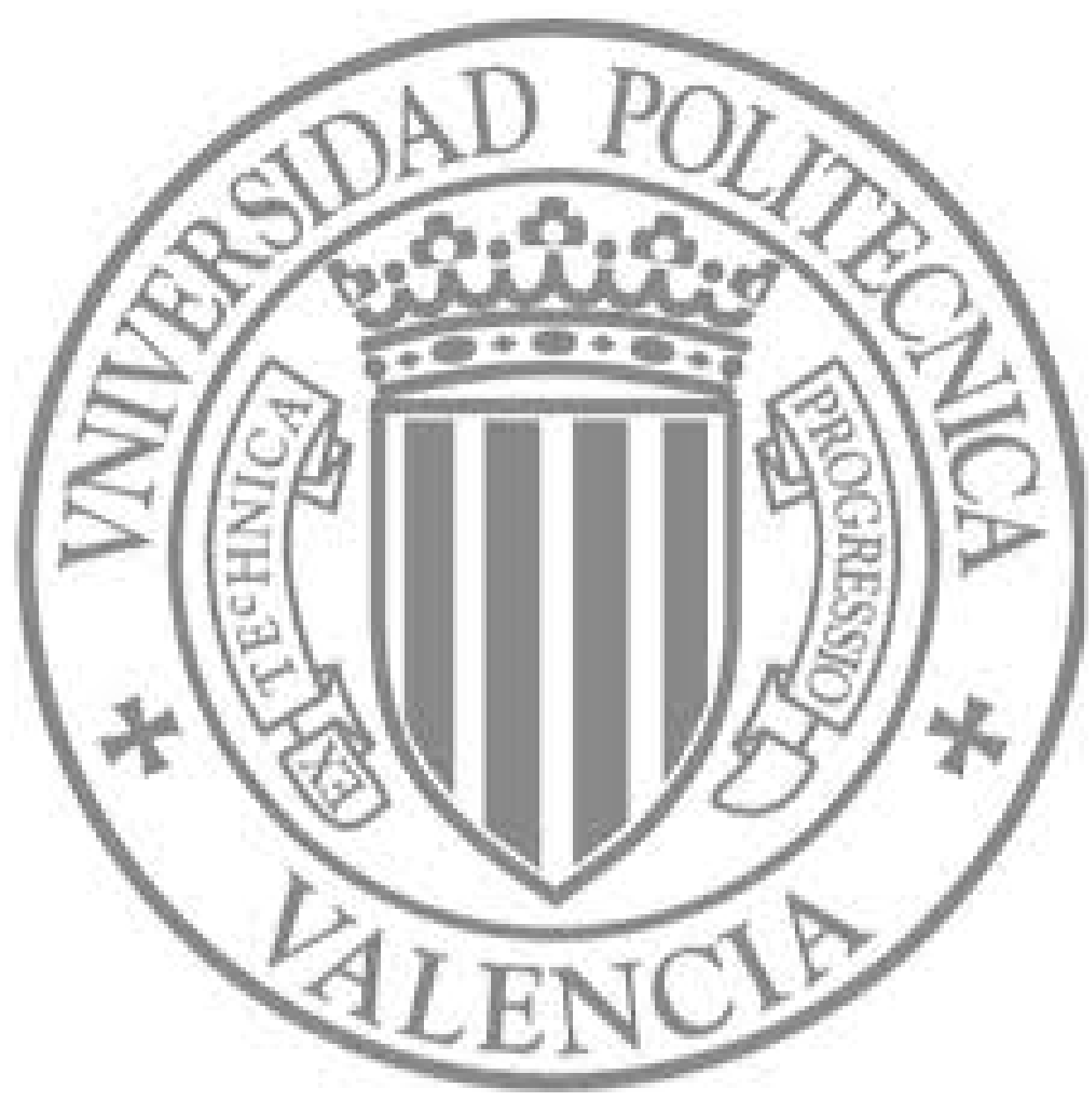

The final publication is available at

http://dx.doi.org/10.1007/s00723-011-0288-3

Copyright Springer Verlag (Germany) 


\title{
Spin chemistry investigation of peculiarities of photoinduced electron transfer in donor-acceptor linked system.
}

I.M. Magin $^{1 *}$, N.E. Polyakov ${ }^{1}$, E.A. Khramtsova ${ }^{1,2}$, A.I. Kruppa ${ }^{1 *}$, A.A Stepanov ${ }^{1}$, P.A. Purtov ${ }^{1}$, T.V. Leshina ${ }^{1}$, Yu.P. Tsentalovich ${ }^{3}$, M.A. Miranda ${ }^{4}$, E. Nuin ${ }^{4}$ and M.L. Marin ${ }^{4}$

${ }^{1}$ Institute of Chemical Kinetics and Combustion SB RAS, Institutskaya 3, Novosibirsk, 630090, Russia

${ }^{2}$ Novosibirsk State University, Pirogova 2, Novosibirsk, 630090, Russia

${ }^{3}$ International Tomography Center SB RAS, Institutskaya 3-a, Novosibirsk, 630090, Russia

${ }^{4}$ Instituto de Tecnologia Quimica, UPV-CSIC, Universidad Politecnica de Valencia, Av. de los

Naranjos s/n, E-46022, Valencia, Spain.

* Corresponding author. E-mail: kruppa@kinetics.nsc.ru, Tel: +7(3833)332947, Fax: +7(3833)307350

\begin{abstract}
.
Photoinduced intramolecular electron transfer (PET) in linked systems, $(R, S)$ - and $(S, S)$ naproxen-N-methylpyrrolidine dyads, has been studied by means of spin chemistry methods (magnetic field effect and chemically induced dynamic nuclear polarization, CIDNP). The relative yield of the triplet state of the dyads in different magnetic field has been measured, and dependencies of the high field CIDNP of N-methylpyrrolidine fragment on solvent polarity have been investigated. However, both $(S, S)$ - and $(R, S)$-diastereoisomers demonstrate almost identical CIDNP effects for the entire range of polarity. It has been demonstrated that the main peculiarities of photoprocesses in this linked system are connected with the participation of singlet exciplex alongside with PET in chromophore excited state quenching.
\end{abstract}

Keywords: spin chemistry, photoinduced electron transfer, donor-acceptor linked systems, exciplex, radical ions 


\section{Introduction}

One of the elementary chemical processes, single electron transfer (SET), has been studied with undiminished interest for many years due to the presence of SET in many key processes important for chemistry and biochemistry, such as photosynthesis, reactions involving certain enzymes (oxygenase, dioxygenase, reductase, cytochromes, peroxidase, etc.), thermal and photoinduced transformations of organic compounds [1-3]. To date, a detailed study of the photoinduced electron transfer processes (PET) has been made, in particular, the effect of factors such as the electronic structure of the donor and the acceptor, the polarity of the medium, and the excitation energy [4]. Less is known about steric factors, in particular, the effect of chiral centers present in the donor or acceptor molecular fragments. Meanwhile, the chiral systems attract special attention in recent years because of the importance of optical isomers for different areas of science, from the origin of life to pharmacological problems related to different activity of drug enantiomers [5-7].

PET processes are investigated by various techniques including laser flash photolysis, Raman spectroscopy, pulse EPR spectroscopy and spin chemistry [7-9]. It is worth noting that in these studies the methods of spin chemistry occupy a special place. Using these methods, SET was proven for the first time in a number of processes, in which it had been only assumed (e.g., nucleophilic substitution reactions and sigmatropic rearrangement), or even not assumed (quenching of the excited states of aromatic donors and electron acceptors, including sensitized cis-trans photoisomerization) $[10,11]$.

In recent years, the peak of interest in studying PET processes has shifted to the so-called linked systems - molecules, which contain both donor and acceptor groups connected by rigid or flexible bridges $[12,13]$. The ultimate goal of such studies is usually announced to be the design of various electronic devices and energy storage systems (light conversion systems such as photosynthetic devices). These studies may also serve as useful models for understanding the processes of electron transfer over long distances, which are postulated for several practically important enzyme systems, for example, cytochrome-cytochrome oxidase [2]. Indeed, linked systems with different rigid or flexible spacers have proven to be good models to study the features of electron transfer processes. Note that most spin chemistry studies of linked systems (dyads and triads) were carried out using only magnetic field effects (MFE) $[12,14]$. The main information obtained from the analysis of magnetic field dependence concerns the energy of the electron exchange interaction between paramagnetic centers of dyads and triads. The dyads containing aromatic chromophores like naphthalene, and amine as a quencher, are classic, well studied examples of the coupled systems [15]. 
Recently the difference in fluorescence quenching rate between $(R, S)$ - and $(S, S)$ enantiomers of naproxen-pyrrolidine dyads (NPX-Pyr) has been observed. This quenching can proceed via the exciplex formation or photoinduced electron transfer, which are typical photoinduced reactions between an excited naphthalene chromophore and electron donors like amine. These two pathways should be considered as the main quenching mechanisms. Other possibilities like hydrogen transfer or energy transfer can be excluded. The electron transfer rate constants for $(S, S)$ - and $(R, S)$-NPX-Pyr have been determined in [15] from the ratio of quantum yield and fluorescence lifetime to be $\mathrm{k}_{\mathrm{e}}=1.8 \times 10^{8}$ and $2.8 \times 10^{8} \mathrm{~s}^{-1}$ for $(S, S)$ - and $(R, S)$ configuration, respectively.

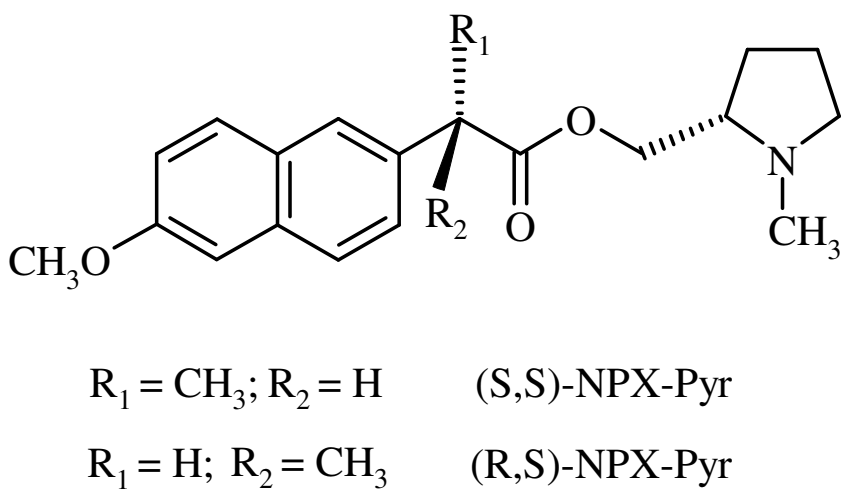

This is the first example of the influence of chirality on the rate of electron transfer we are aware of, although it should be noted that the difference in the rates of fluorescence quenching of various enantiomers is a reliably established fact [16]. The largest effects are obtained in nonpolar media, and generally relate to processes in exciplexes [17]. Indeed, in this case one can expect a stronger influence of the spatial orientation of the partners on the efficiency of quenching. The suggestion that the main mechanism of fluorescence quenching in NPX-Pyr dyad is intramolecular PET has been made from the analysis of thermodynamics parameters of the dyads [15].

The main goal of this study is the investigation of peculiarities of PET in linked systems, $(S, S)$ - and $(R, S)$-NPX-Pyr dyads, by means of MFE and CIDNP (chemically induced dynamic nuclear polarization) techniques. We are going to compare CIDNP effects detected in the NPXPyr dyad with similar results observed in related systems in solution. The systems that were selected for comparison are photoinduced interaction of naphthalene with triethylamine (TEA), methyl ester of naproxen (Me-NPX) with TEA, methyl ester of naproxen with acetate of $(S)-N$ methyl-2-pyrrolidinemethanol (Ac-Pyr). 
<smiles>COC(=O)C(C)c1ccc2cc(OC)ccc2c1</smiles>

Me-NPX<smiles>CC(=O)OC[C@@H]1CCCN1C</smiles>

Ac-Pyr

The first stage of this study is to establish detailed mechanisms of the processes responsible for degradation of the photoexcitation of the dyad using the two methods of spin chemistry, MFE and CIDNP. Analysis of spin polarization in this case seems quite promising, for example, to establishing the roles of biradical ion and exciplex in quenching processes of photoexcited state of the dyad.

\section{Experimental}

The $R, S$ and $S, S$ naproxen-pyrrolidine dyads $((S)$ - $N$-methyl-2-pyrrolidinemethyl $2(R)$ or (S)-(6-methoxy-2-naphthylpropanoate) and the acetate of (S)-N-methyl-2-pyrrolidinemethanol were synthesized as described earlier [16]. Methyl 6-methoxy-2-naphthylpropanoate was synthesized by the same method as described for dyads and was purified by TLC. 2,6-Dimethylnaphthalene (DMN) (Aldrich) was sublimated prior to use. Triethylamine was distilled over zinc powder. Deuteroacetonitrile (D99.9\%) and deuterobenzene (D99.8\%) (both by Aldrich) were used for NMR and CIDNP experiments. Acetonitrile freshly distilled over $\mathrm{P}_{2} \mathrm{O}_{5}$ was used for magnetic field effect experiments.

${ }^{1} \mathrm{H}$ NMR spectra were recorded at room temperature on a DPX-200 BRUKER NMR spectrometer $\left(200 \mathrm{MHz}{ }^{1} \mathrm{H}\right.$ operating frequency, $\left(\tau(90)^{\circ}=5.8 \mu \mathrm{s}\right)$. A Lambda Physik EMG 101 MSC eximer laser was used as the light source $(308 \mathrm{~nm}, 100 \mathrm{~mJ}$ at output window, $20 \mathrm{~mJ} / \mathrm{pulse}$ in sample volume, with pulse duration of $15 \mathrm{~ns}$ ). For time resolved (TR) CIDNP experiments $[18,19]$, the delay between the laser and detection pulses was varied from 0 to $100 \mu$ s. For CIDNP dependencies on solvent polarity the delay was set at $1 \mu \mathrm{s}$. Pseudo steady-state (PSS) photo-CIDNP [20] experiments were performed at room temperature. The samples in standard 5 mm Pyrex NMR tubes were irradiated directly in the probe of NMR spectrometer at $20^{\circ} \mathrm{C}$.

Magnetic field effect (MFE) experiments were performed using a laser flash photolysis apparatus described earlier $\left(\lambda_{e x}=266 \mathrm{~nm}, \lambda_{o b s}=440 \mathrm{~nm}\right.$, pulse length $10 \mathrm{~ns}$, energy $\left.12 \mathrm{~mJ}\right)$ [21]. MFE was monitored at 100 ns delay where the transient absorption reaches the plateau.

Dyad concentration in MFE experiments was $0.2 \mathrm{mM}$ in acetonitrile. For CIDNP experiments the concentrations of dyad, DMN and Me-NPX were $1 \mathrm{mM}$. For experiments in the presence of TEA or Ac-Pyr their concentrations were $10 \mathrm{mM}$. A mixture of deuterobenzene and 
deuteroacetonitrile was used for solvent polarity variation. All samples were bubbled with argon for 15 min to remove dissolved oxygen just before photolysis.

\section{Results and discussion.}

According to the declared goals of this investigation we attempt to confirm the suggestion made in $[16,22]$ that the main way of triplet state formation of the NPX-Pyr dyad in acetonitrile is singlet-triplet conversion in biradical ion - chain linked radical ion pair, see Scheme 1.

Scheme 1. Intramolecular electron transfer in NPX-Pyr dyad.

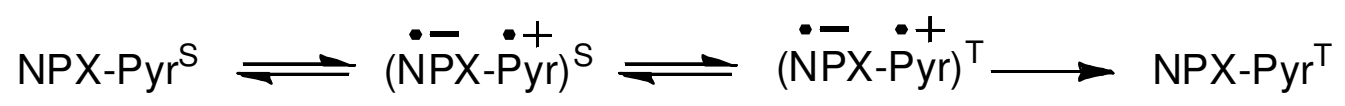

Since the proposed way of triplet state formation includes singlet-triplet conversion in radical ion pair (RIP), and the rate constant of this stage depends on external magnetic field, first we tried to measure magnetic field effect to check Scheme 1.

Several mechanisms of the influence of external magnetic field on the process of photoinduced electron transfer in this system can be expected. In the case of weak electron exchange interaction between the donor and acceptor fragments of the biradical ion (as compared to hyperfine interaction (HFI) constants), the usual HFI- and $\Delta \mathrm{g}$-mechanisms must be involved in spin evolution [11]. In the case of strong electron exchange interaction other mechanisms are possible: electron relaxation induced spin state transitions [23] and the level-crossing (J) mechanism [24]. In the latter case the dependence of the triplet yield on external magnetic field will correlate with the value of the average electron exchange coupling in the radical ion pair $\left(\mathrm{H}_{\max } \sim 2 \mathrm{~J}_{0}\right)$. The majority of experimentally observed values of $\mathrm{J}_{0}$ are in the range of up to $1 \mathrm{~T}$ $[12,14,25,26]$. The values of HFI constants and the difference of g-factors of the donor and acceptor fragments in discussed biradical ion let us also expect the appearance of MFE formed via the HFI and relaxation mechanisms [11].

To imagine the possible spatial configurations of the dyads and a possible variation of the distance between the donor and acceptor groups, which will determine the energy of the electron exchange interaction in the biradical ion, we have examined the calculated energy variation with the torsion angle of $\mathrm{CH}_{3}-\mathrm{C}-\mathrm{C}=\mathrm{O}$ bond of $(\mathrm{R}, \mathrm{S})$ - and $(\mathrm{S}, \mathrm{S})-\mathrm{NPX}-\mathrm{Pyr}$ (Figure 1). These figures show that the distances between the donor and acceptor groups of the dyads in the energy minima differ significantly for $(R, S)$ - and $(S, S)$-diastereoisomers. 

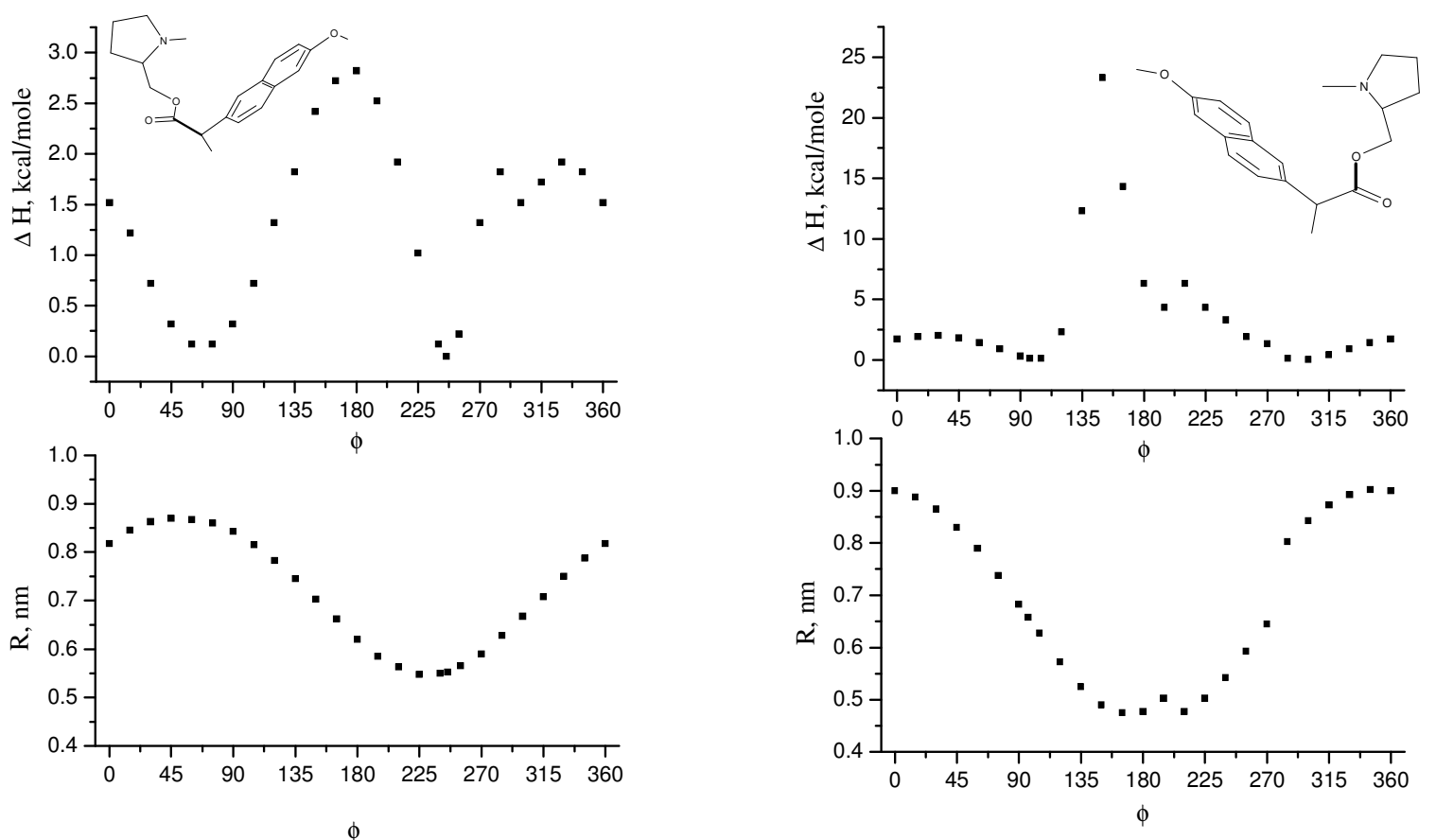

Figure 1. Calculated changes in the heat of formation and the distance between the donor and acceptor groups of a) (R,S)-NPX-Pyr and b) (S,S)-NPX-Pyr as a function of the torsion angle ( $\phi$ ) of $\mathrm{CH}_{3}-\mathrm{C}-\mathrm{C}=\mathrm{O}$ bond.

In this case the minimum distance between the donor and acceptor groups (the nitrogen atom and the center of the naproxen ring) is around $0.5-0.55 \mathrm{~nm}$, and the maximum distance is about 0.8-0.9 $\mathrm{nm}$. On the other hand, it is well known that biradicals of cyclic ketones having chain length of 10 carbon atoms have maximum of CIDNP in magnetic field around $100 \mathrm{mT}$. It could be expected that the maximum of MFE in the system under study will be in an even lower magnetic field because of the more rigid structure expected for the biradical ion of the dyad. Taking into account all these considerations, magnetic field strength in this investigation was varied from 0 to $70 \mathrm{mT}$.

MFE on the yield of triplet state of the dyad was measured using laser flash photolysis. An example of the triplet decay kinetics for NPX-Pyr is shown in Figure 2. Magnetic field dependence of the triplet yield was measured at the maximum of these curves. Unfortunately, no observable magnetic field effect was detected in this system (Figure 3). 


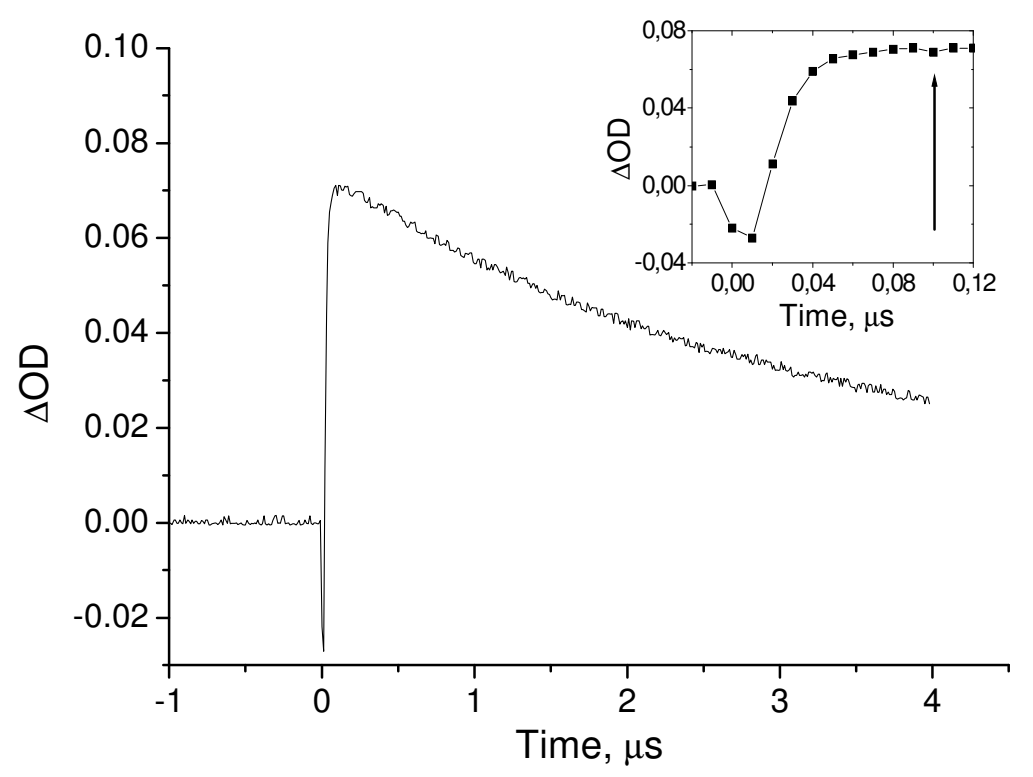

Figure 2. Kinetics of transient absorption of $(R, S)-N P X-P y r$ triplet state detected after laser pulse $\left(\lambda_{o b s}=440 \mathrm{~nm}\right)$. Inset shows the initial part of the kinetics. Arrow indicates time delay for monitoring of MFE.

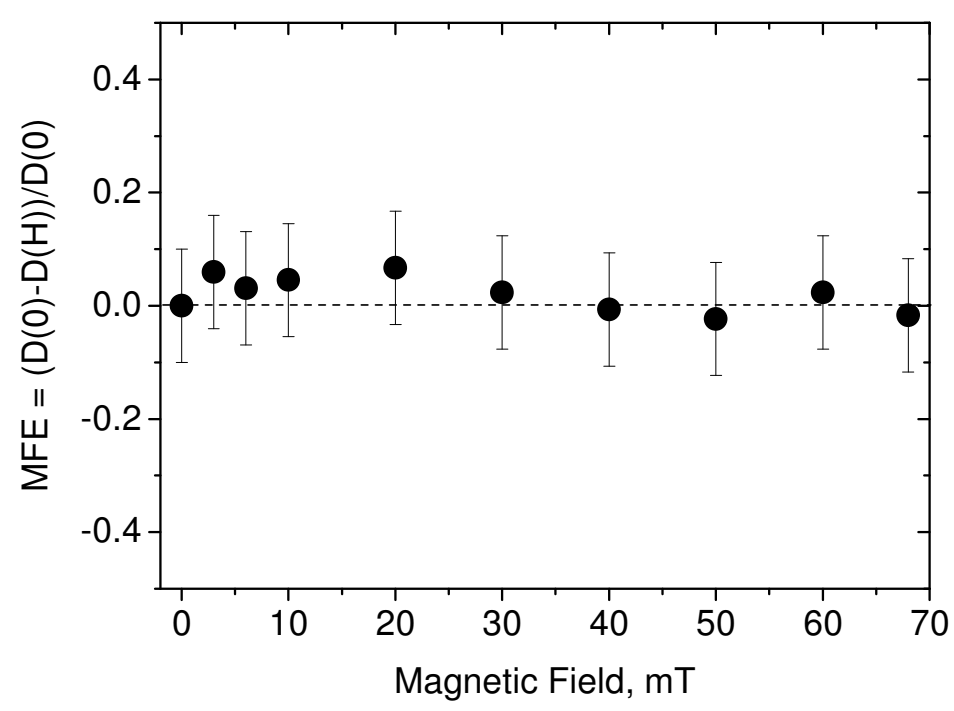

Figure 3. Magnetic field dependence of the yield of (R,S)-NPX-Pyr triplet state in acetonitrile measured by laser flash photolysis.

The reason for the absence of the observable MFE might be the wrong choice of the range of magnetic fields. This is possible if the energy of electron exchange interaction in the biradical ion is much higher than the value of the applied external magnetic field. According to our calculations [27], in a radical pair with $\mathrm{J}>>$ A (HFI constant) the absence of MFE formed through the HFI mechanism is also possible. On the other hand, the very rapid growth of the triplet yield, with the characteristic time about 20 ns (see insert in Figure 2), casts doubts on the possibility of the PET stage as the main route of the triplet state formation. Note that both PET 
and exciplex formation are typical photoinduced processes, which can be expected to occur between excited naphthalene chromophores and electron donors like amines [15]. These two pathways should be considered as the main quenching mechanisms. The formation of trace amounts of exciplex during UV irradiation of the dyads in acetonitrile was described earlier [16]. Calculations show that exciplex formation is favored in non-polar and weakly-polar solvents, while in polar media photoinduced electron transfer has to dominate (Figure 4). These calculations were performed using the Rehm-Weller equation $\Delta \mathrm{G}(\varepsilon)=\left[\mathrm{E}_{\mathrm{ox}}\left(\mathrm{D} / \mathrm{D}^{+}\right)-\mathrm{E}_{\mathrm{red}}\left(\mathrm{A}^{-} / \mathrm{A}\right)\right]_{\varepsilon 0}$ $-\mathrm{e} / \varepsilon a+\Delta \mathrm{G}_{\text {solv }}-\mathrm{E}_{0-0}\left({ }^{1} \mathrm{D}^{*}\right)$. Here $\Delta \mathrm{G}_{\mathrm{solv}}=\mathrm{e}^{2} / 2\left(1 / \mathrm{R}^{\mathrm{D}+}+1 / \mathrm{R}^{\mathrm{A}-}\right) \times\left(1 / \varepsilon-1 / \varepsilon_{0}\right), \varepsilon_{0}$ is dielectric constant of the solvent, in which redox potentials were determined; $\mathrm{R}^{\mathrm{D}+}, \mathrm{R}^{\mathrm{A}-}$ and $a$ are the radii of the radical cation, radical anion, and the distance between them, respectively. $\mathrm{E}_{\mathrm{ox}}(\mathrm{N}$ methylpyrrolidine $=1.0 \mathrm{~V}$ vs. SCE, $\mathrm{E}_{\mathrm{red}}(2-$ methoxynaphthalene $)=-2.6 \mathrm{~V}$ vs. SCE were determined in acetonitrile [28], and $\mathrm{E}_{0-0}(\mathrm{NPX})=3.69 \mathrm{eV}$.

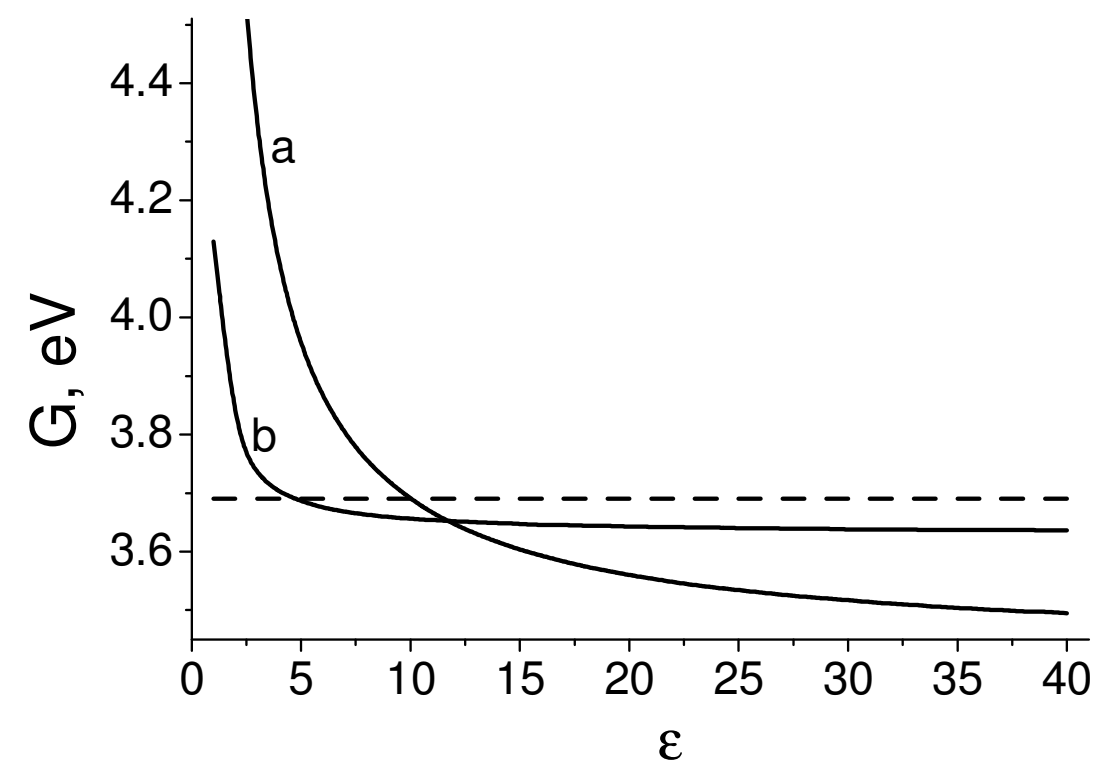

Figure 4. Dependencies of the free energy of NPX-Pyr biradical ion (a) and exciplex (b) on dielectric constant of solvent $(\varepsilon)$. Dashed line shows the energy of excited singlet state of NPXPyr.

To verify the participation of PET stage in the quenching of NPX-Pyr excited state we use another spin chemistry method - high field CIDNP. Figure 5 shows NMR and CIDNP spectra of $(S, S)$-NPX-Pyr in acetonitrile. The $(R, S)$-diasstereoisomer demonstrates identical CIDNP pattern. Noticeable polarization was detected only for pyrrolidine protons in $\alpha$-position to $\mathrm{N}$ atom. It is reasonable to suggest that this polarization is derived from the biradical ion, because HFI constants of amine radical cation are maximal on the $\mathrm{N}-\mathrm{CH}_{3}, \mathrm{~N}-\mathrm{CH}_{2}$ and $\mathrm{N}-\mathrm{CH}$ groups in the system under study [29]. 


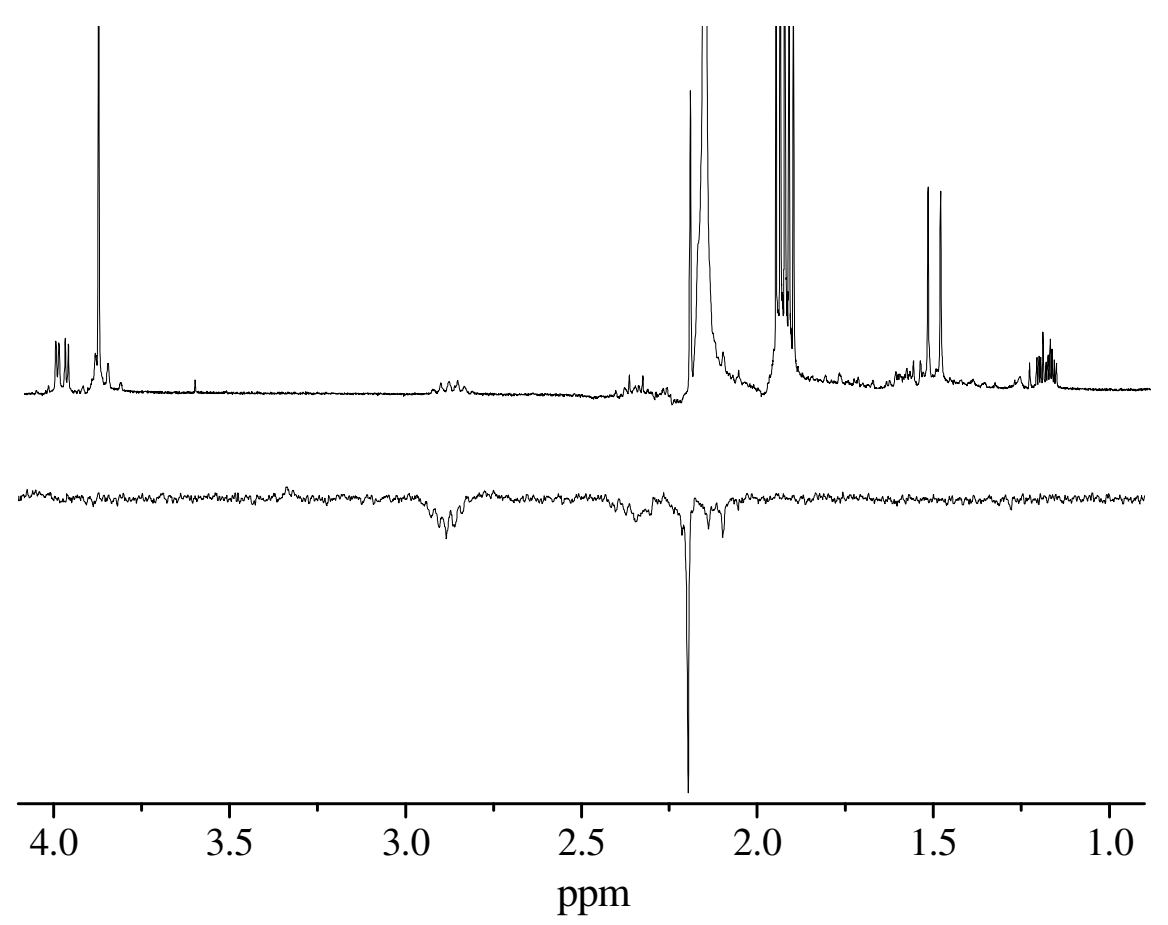

Figure 5. NMR (top) and TR CIDNP at l $1 \mu$ s delay (bottom) spectra detected after laser irradiation of $(S, S)-N P X-P y r(1 \mathrm{mM})$ in $C D_{3} C N$.

Hence, it makes sense to assume that this polarization is formed through the $\mathrm{S}-\mathrm{T}_{0}$ mechanism in accordance with the results of numerous previous investigations of chemical polarization formed in short biradicals in high magnetic field during the photolysis of cyclic ketones [11] and benzoquinone in the presence of quadricyclane [30]. In the framework of the S$\mathrm{T}_{0}$ mechanism the appearance of chemical polarization of the NPX-Pyr dyad would be possible if there is some other channel of nuclear polarization decay in addition to back electron transfer in the biradical ion. We suppose it might be the processes of fast relaxation in the biradical, which is usually responsible for the relaxation mechanism of MFE [11].

Since the indicative feature of CIDNP formed in radical ion pairs is their high sensitivity to the solvent polarity, the dependence of chemical polarization of $\mathrm{N}$-methylpyrrolidine protons on dielectric constant of the solvent $(\varepsilon)$ has been investigated to clarify the role of the PET channel in the quenching of excited state of the NPX-Pyr dyad. Note that CIDNP effects observed for this dyad have to be a combined result of several contributions, namely, the recombination of $\mathrm{S}$ and $\mathrm{T}$ states of the biradical ion (intramolecular back electron transfer), the bimolecular quenching of the excited state of the chromophore of the dyad by another dyad in ground state, as well as the bulk processes (degenerate electron exchange of the radical ions with neutral molecules and F-pairs). To minimize the contribution from intermolecular processes to the observed CIDNP, low concentration of the dyad $(\leq 1 \mathrm{mM})$ was used in our experiments. Besides, TEA was added as an additional external quencher of the excited state of the naproxen fragment. 
In this case the rate of intramolecular quenching of the excited singlet state of the dyad would significantly exceed the rates of intermolecular processes: $\mathrm{W}_{\text {intra }}=1.8 \times 10^{8} \mathrm{~s}^{-1}$ and $2.8 \times 10^{8} \mathrm{~s}^{-1}$, for $(S, S)$ - and $(R, S)$-NPX-Pyr, respectively; $\mathrm{W}_{\mathrm{TEA}}=5 \mathrm{x} 10^{9} \mathrm{x}[\mathrm{TEA}]=5 \times 10^{7} \mathrm{~s}^{-1}$ and $\mathrm{W}_{\text {inter }}=5 \mathrm{x}$ $10^{9} \times[\mathrm{dyad}]=5 \times 10^{6} \mathrm{~s}^{-1}$, for the used experimental concentrations and assuming that the rate constants of the quenching of the excited state of the dyad by the dyad itself and by amine are equal [15]. Thus, we suppose that all experimental dependencies of NPX dyad CIDNP that will be described later refer to intramolecular PET.

Experimental dependencies of the TR CIDNP intensity for $\mathrm{N}$-methylpyrrolidine protons $\left(\mathrm{N}-\mathrm{CH}_{3}\right.$ and $\left.\mathrm{N}-\mathrm{CH}\right)$ and $\mathrm{N}-\mathrm{CH}_{2}$ protons of TEA on solvent dielectric constant $(\varepsilon)$ are shown in Figure 6. Dependencies of the CIDNP intensities for $\mathrm{CH}_{2}$ protons of TEA and $\mathrm{N}-\mathrm{CH}_{3}$ protons of $\mathrm{N}$-methylpyrrolidine on solvent polarity in the systems DMN + TEA, Me-NPX + TEA and MeNPX + Ac-Pyr are shown in Figures 7 and 8. All these systems have very close values of redox potentials in acetonitrile: $\mathrm{E}_{\mathrm{red}}=2.60 \mathrm{~V}$ vs. $\mathrm{SCE}$ for methoxynaphthalene; $\mathrm{E}_{\mathrm{red}}=2.58 \mathrm{~V}$ vs. $\mathrm{SCE}$ for DMN; $\mathrm{E}_{\mathrm{ox}}=0.96 \mathrm{~V}$ vs. SCE for TEA and $\mathrm{E}_{\mathrm{ox}}=1.0 \mathrm{~V}$ vs. SCE for N-methylpyrrolidine [28].

Note that dependencies of the CIDNP effects for protons of the $\mathrm{N}$-methylpyrrolidine fragment and TEA detected during UV irradiation of NPX-Pyr dyad differ significantly from the profiles detected in photoinduced interaction of Ac-Pyr and TEA with naphthalene derivatives. In particular, at high solvent polarity the sign of CIDNP for Ac-Pyr (Figure 8b) or TEA (Figures $6 \mathrm{~b}, 7,8 \mathrm{a}$ ) is opposite to that for protons of the N-methylpyrrolidine fragment of the dyad (Figure 6). Moreover, only in the photolysis of the dyad (Figure 6) and Me-NPX and Ac-Pyr (Figure 8b) is CIDNP observed at $\varepsilon<10$.

a

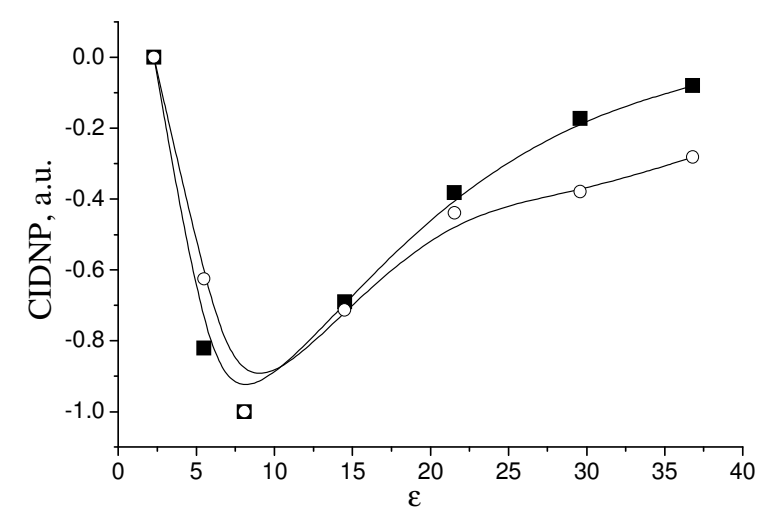

$\mathrm{b}$

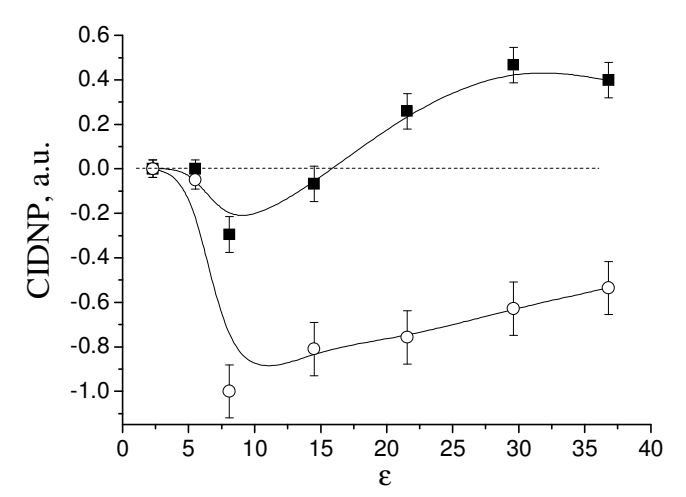

Figure 6. Dependencies of the TR CIDNP (delay $1 \mu$ s) intensity for $\mathrm{N}-\mathrm{CH}_{3}$ protons of NPX-Pyr dyad (1 mM) on solvent polarity in the absence ( $a$, left: $(\mathbf{\square})-(R, S)-$ and $(0)-(S, S)$-dyad) and in the presence of TEA (10mM) (b, right: (०)- $(\mathrm{R}, \mathrm{S})$-dyad and (ם)- $\mathrm{N}-\mathrm{CH}_{2}$ protons of TEA). 


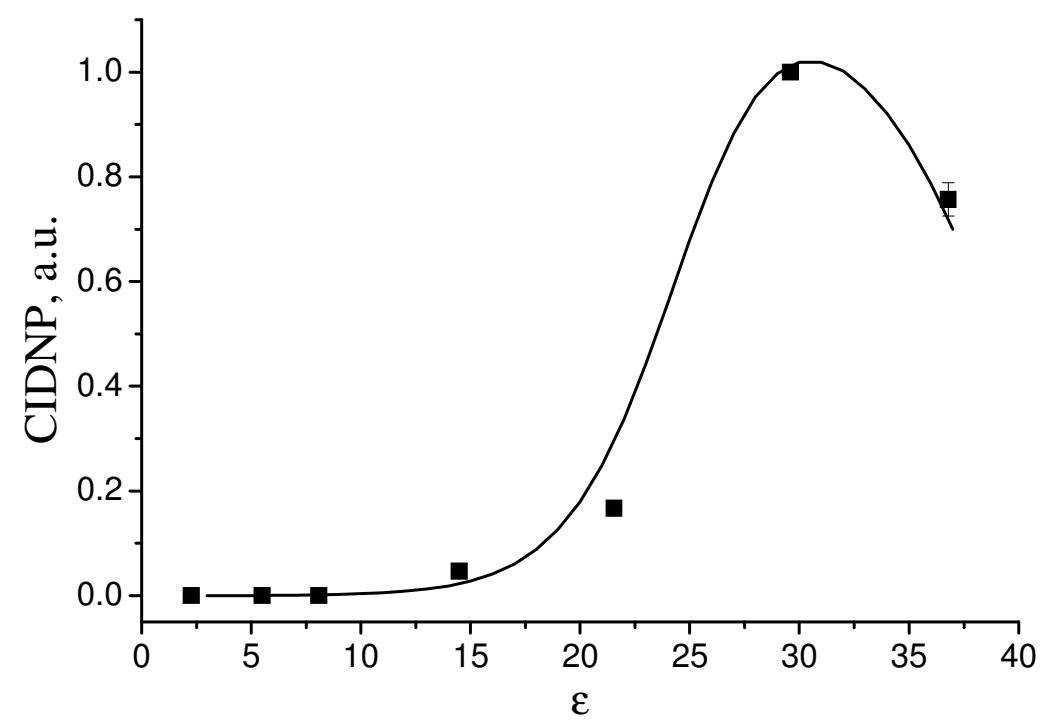

Figure 7. Dependence of the TR CIDNP (delay $1 \mu \mathrm{s})$ intensity for $\mathrm{N}-\mathrm{CH}_{2}$ protons of TEA on solvent polarity in its photoreaction with DMN.

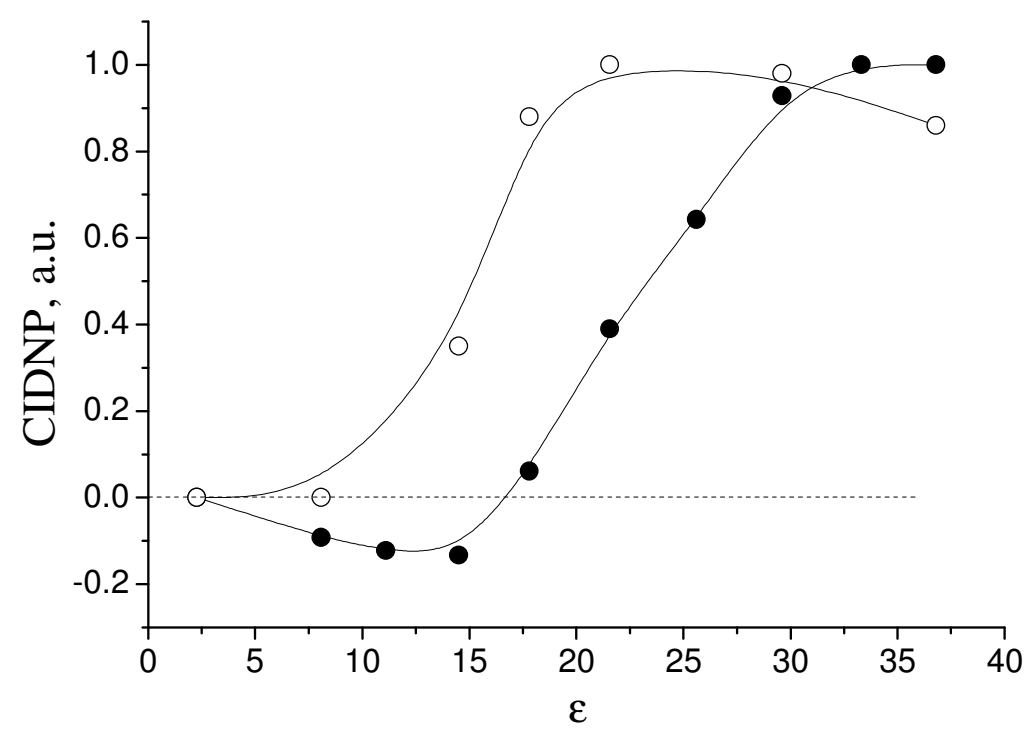

Figure 8. Dependencies of the TR CIDNP (delay 1us) intensity on solvent polarity in the systems: a) Me-NPX and TEA (O); b) Me-NPX and Ac-Pyr (•).

Furthermore, the dependencies for $\mathrm{N}-\mathrm{CH}_{2}$ protons of TEA detected in photoinduced interaction of TEA with the dyad (Figure 6b) and $\mathrm{N}_{-} \mathrm{CH}_{3}$ protons of Ac-Pyr in its interaction with Me-NPX show the alternation of the signs of CIDNP (Figure 8b).

To explain the unusual CIDNP dependencies on solvent polarity we turned to reference data. According to classical considerations, the S-type curve is the typical shape of the dependence of the quantum yields of solvent separated RIP on solvent dielectric constant [31]. It is also known that charge transfer complexes, for instance, the complex of stilbene with fumaronitrile, under UV irradiation form radical cations of stilbene even in solvents with low 
polarity, bromobenzene and dimethoxyethane [32]. The authors suggested that the presence of the radical cation in nonpolar solvents is due to fast, comparable with lifetime of RIP, transitions between solvent separated and contact RIP (exciplex). The observation of MFE on exciplex fluorescence in chain linked phenanthrene-dimethylaniline dyad and in supramolecular complex of zinc(II)-tetraphenylporphyrin with viologen, which was described by Japanese researchers [33], was also explained by the existence of fast equilibrium between RIP and exciplex.

Because the CIDNP intensity in the systems under study in different solvents is mostly determined by RIP concentration, for radical ions with redox potentials close to the values for NPX and Pyr significant effects should appear at polarity around $\varepsilon=15-20$. Examples are CIDNP effects for TEA protons in systems DMN+TEA and Me-NPX+TEA detected in solvents with different polarity (Figure 7 and 8a). Equal CIDNP signs for these protons point to the same predominant channel of RIP recombination in both systems. Note that T-RIP recombination is the main channel of the photoinduced interaction of naphtalene with TEA in polar solvents, as was established earlier [29]. It is well known that the sign of the geminal CIDNP that is formed in RIP is determined by the ratio of the recombination probabilities for $\operatorname{singlet}\left(\lambda_{S}=\frac{k_{S} \tau}{1+k_{S} \tau}\right)$ and triplet RIP $\left(\lambda_{T}=\frac{k_{T} \tau}{1+k_{T} \tau}\right)$, where $\mathrm{k}_{\mathrm{S}}$ and $\mathrm{k}_{\mathrm{T}}$ are recombination rate constants and $\tau$ is RIP lifetime [34]. Thus, $\lambda_{\mathrm{T}}>\lambda_{\mathrm{S}}$ holds for the abovementioned interaction of DMN and Me-NPX with TEA. The participation of Ac-Pyr, instead of TEA, in reaction with Me-NPX changes dramatically the shape of the CIDNP dependence on polarity (Figure 8). Thus, the triplet recombination channel plays the dominant role in the solvents with high polarity $(\varepsilon>20)$. A decrease of polarity down to $\varepsilon=15$ reduces CIDNP intensity, and further decreasing of the dielectric constant leads to the change of sign of CIDNP for $\mathrm{N}-\mathrm{CH}_{3}$ protons. The change of sign most probably means that in this case $\lambda_{\mathrm{S}}$ becomes larger than $\lambda_{\mathrm{T}}$. This should be expected if $\mathrm{S}$ state of RIP converts into exciplex. Analysis of CIDNP for protons of the pyrrolidine fragment of the dyad shows that $\lambda_{\mathrm{T}}<$ $\lambda_{\mathrm{S}}$ in the entire polarity range. If this results from singlet exciplex formation, further decrease of CIDNP intensity with the increase of polarity (Figure 6) might be explained by the change of the ratio of $\lambda_{\mathrm{S}}$ and $\lambda_{\mathrm{T}}$ arising from the increase of the triplet channel recombination in polar media. Additional evidence for the formation of exciplex of the NPX-Pyr dyad is provided by the profile of the CIDNP dependence on polarity measured in the photoinduced reaction of the NPX-Pyr dyad with TEA. While CIDNP for methyl protons of the pyrrolidine fragment is identical to that detected in the absence of TEA and corresponds to intramolecular PET, CIDNP effects for N$\mathrm{CH}_{2}$ protons of TEA, which are polarized through intermolecular quenching process, demonstrate the abovementioned peculiarities (Figure 6b). A comparison of the polarity 
dependencies of CIDNP for TEA protons detected in quenching of the excited state of the NPXPyr dyad and Me-NPX itself by TEA (Figure $6 \mathrm{~b}$ and Figure 8a) points to the participation of the exciplex of the dyad in the reaction with TEA. Because the same paramagnetic species are formed in both reactions, the reason for the appearance of CIDNP in solvents with low polarity $(5<\varepsilon<15)$ and the change of the dominant recombination channel in high polarity $\left(\lambda_{\mathrm{T}}<\lambda_{\mathrm{S}}\right)$ most probably might be connected with the singlet exciplex formation. Possible ways for quenching of the excited singlet state of the NPX-Pyr dyad in the presence and in the absence of TEA are shown below.

Scheme 2. Quenching of the excited singlet state of the NPX-Pyr dyad in solution.

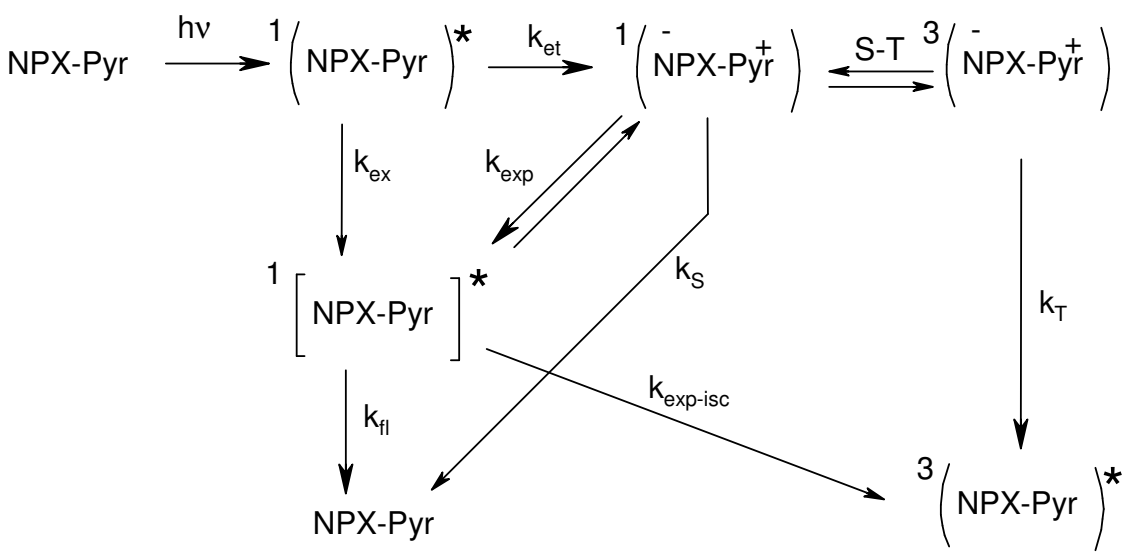

Scheme 3. Quenching of the excited singlet state of the NPX-Pyr dyad in solution in the presence of TEA.

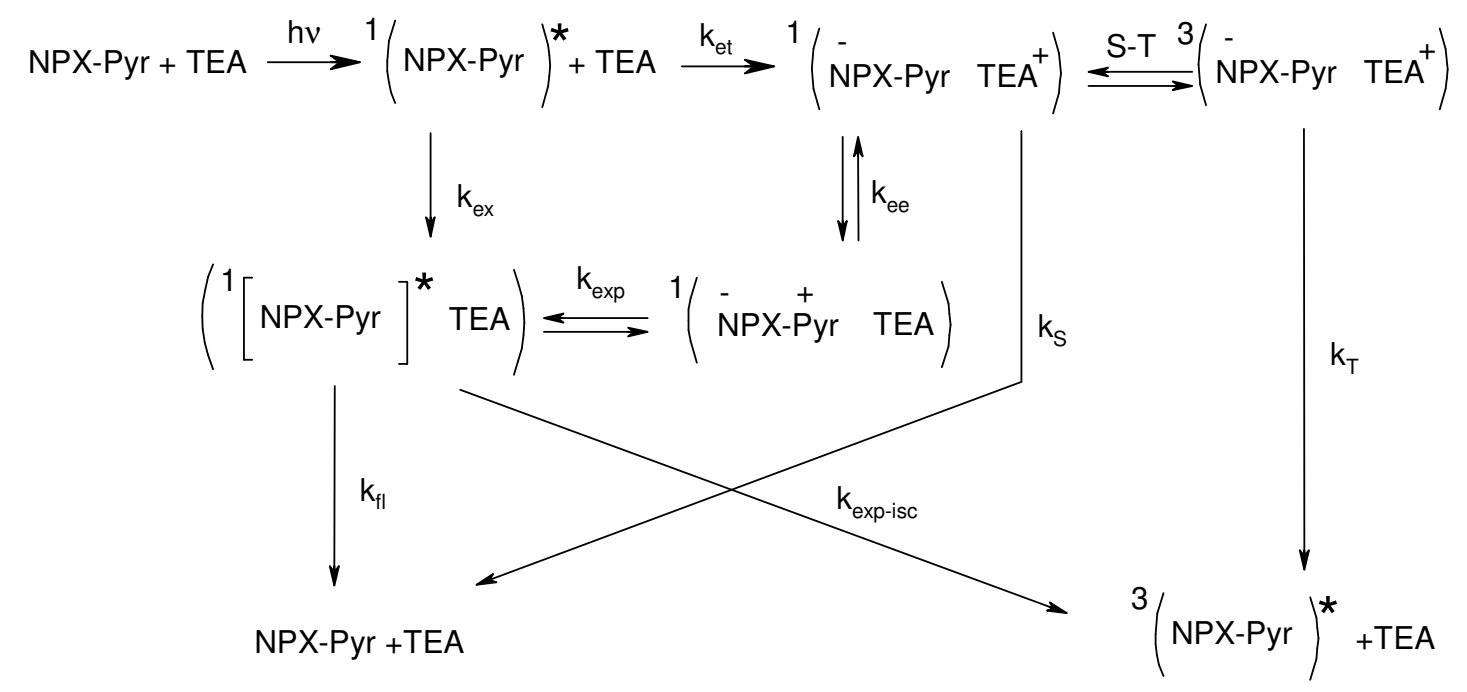

These schemes reflect the main peculiarity of the system under study connected with the existence of fast, comparable with the time of spin evolution $\left(K_{\mathrm{S}-\mathrm{T}}\right)$, equilibrium between the exciplex and the biradical ion $\left(K_{\text {exp }}\right)$ or RIP that are formed in the presence of TEA $\left(\mathrm{k}_{\mathrm{exp}}\right.$ and $\left.\mathrm{k}_{\mathrm{ee}}\right)$. 
To explain the appearance of CIDNP for TEA protons in weakly polar media $(\varepsilon<10)$ the step of electron transfer from the pyrrolidine fragment of the dyad to TEA has been included in scheme 3. Since the difference of oxidation potentials of TEA and the pyrrolidine fragment of the dyad is less than $0.05 \mathrm{~V}$, it is reasonable to expect rapid electron exchange between the radical cation of TEA and the pyrrolidine fragment of the dyad ( $k_{e e}$ in sheme 3$)$. Due to charge separation in the biradical ion of the dyad the electron exchange should lead to formation of the initial charge separated RIP of the radical anion of the dyad and the radical cation of TEA (-NPX-Pyr TEA ${ }^{+}$). It is this initial charge separation in RIP that is the origin of the CIDNP effects for TEA protons in solvents with low polarity.

According to these schemes, the signs of the observed CIDNP are determined by the ratios of recombination constants $\mathrm{k}_{\mathrm{S}}, \mathrm{k}_{\mathrm{T}}$ and $\mathrm{k}_{\exp }$. Correspondingly, the recombination probabilities for $\mathrm{S}$ and T spin states of RIP are given by the following equations: $\lambda_{T}=\frac{k_{T} \tau}{1+k_{T} \tau}, \lambda_{S}=\frac{\left(k_{S}+k_{\exp }\right) \tau}{1+\left(k_{S}+k_{\exp }\right) \tau}$.

Analysis of energy diagrams for the photoinduced processes in the NPX-Pyr dyad in the presence of TEA in the media of different polarity leads to the conclusion that the change of the CIDNP sign for TEA is possible under the following conditions (Figure 9).
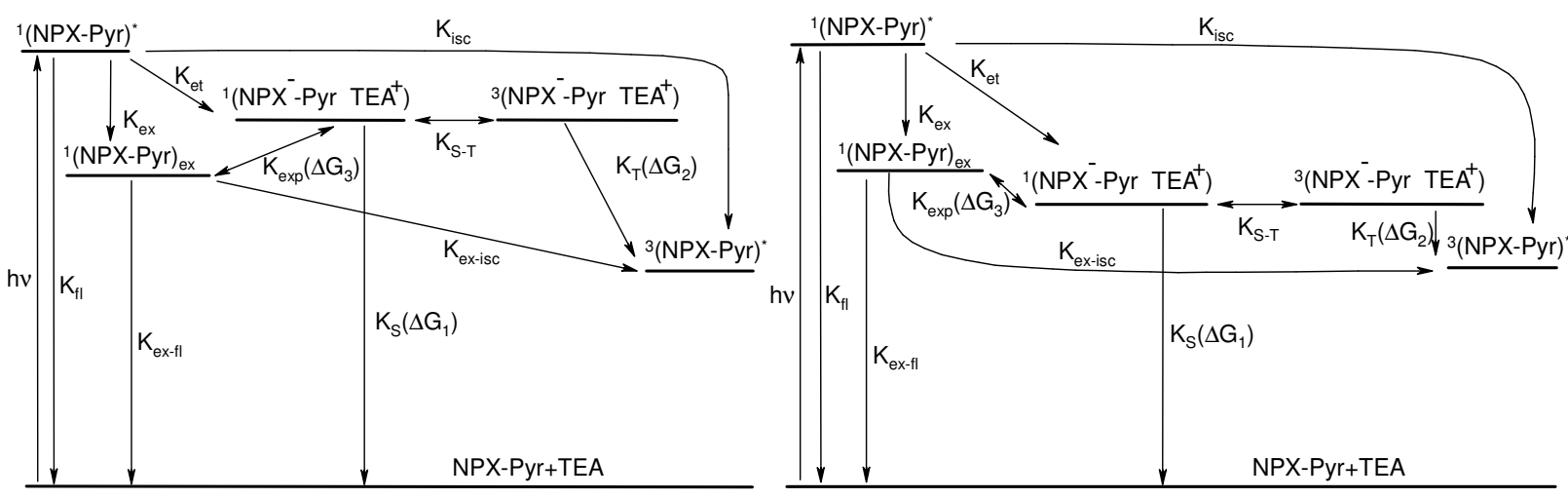

Figure 9. Energy diagrams for the photoinduced processes in the NPX-Pyr dyad in the presence of TEA: (left) - low polarity, (right) - high polarity.

According to Figure 9, the ratios of $\mathrm{k}_{\mathrm{exp}}, \mathrm{k}_{\mathrm{S}}$ and $\mathrm{k}_{\mathrm{T}}$ in media of different polarity depend on the free energy changes $\Delta \mathrm{G}_{3}, \Delta \mathrm{G}_{1}$ and $\Delta \mathrm{G}_{2}$, correspondingly. One can expect that at low polarity $(\varepsilon<20) \Delta \mathrm{G}_{1}, \Delta \mathrm{G}_{2}, \Delta \mathrm{G}_{3}<0$ and $\left|\Delta \mathrm{G}_{3}\right|<\left|\Delta \mathrm{G}_{2}\right|<\left|\Delta \mathrm{G}_{1}\right|$. In this case the expected ordering of the rate constants will be $\mathrm{k}_{\exp }>\mathrm{k}_{\mathrm{T}}>\mathrm{k}_{\mathrm{S}}$, and the sign of CIDNP for the TEA protons corresponds to back electron transfer from S-RIP $\left(\lambda_{\mathrm{S}}>\lambda_{\mathrm{T}}\right)$. On the other hand, at higher polarity $(\varepsilon>20)$ one can expect that $\Delta \mathrm{G}_{1}, \Delta \mathrm{G}_{2}<0$ and $\Delta \mathrm{G}_{3}>0$ and $\left|\Delta \mathrm{G}_{2}\right|<\left|\Delta \mathrm{G}_{1}\right|$. Then $\mathrm{k}_{\mathrm{T}}>\mathrm{k}_{\mathrm{S}}>\mathrm{k}_{\mathrm{exp}}$, and the sign of CIDNP for the TEA protons will correspond to $\lambda_{\mathrm{T}}>\lambda_{\mathrm{S}}$. Thus, the observed CIDNP effects and their dependence on solvent polarity can be explained only by including exciplex (existing in equilibrium with RIP, $K_{\text {exp }} \sim K_{\text {S-T }}$ ) into the reaction scheme. 
Thus, analysis of the dependencies of CIDNP intensity on solvent polarity points to the participation of singlet exciplex in quenching of the NPX excited state in the following three systems: NPX-Pyr dyad in the presence and in the absence of TEA, and Me-NPX in the presence of Ac-Pyr. Reference data is also available on the detection of exciplex of NPX with Nmethylpyrrolidine by optical methods [15]. CIDNP polarity dependencies for these systems manifest both a similarity, the existence of a region where $\lambda_{S}>\lambda_{\mathrm{T}}$, and a difference, in the region of high polarity. So, CIDNP analysis shows the domination of the singlet recombination channel up to solvent dielectric constant $\varepsilon=20$ in the case of quenching of the excited states of Me-NPX in solution, whereas for the process of quenching of the excited state of the NPX-Pyr dyad $\lambda_{S}>\lambda_{T}$ in the entire range of solvent polarity. The latter means that dyad quenching through the exciplex competes with PET even in polar solvents.

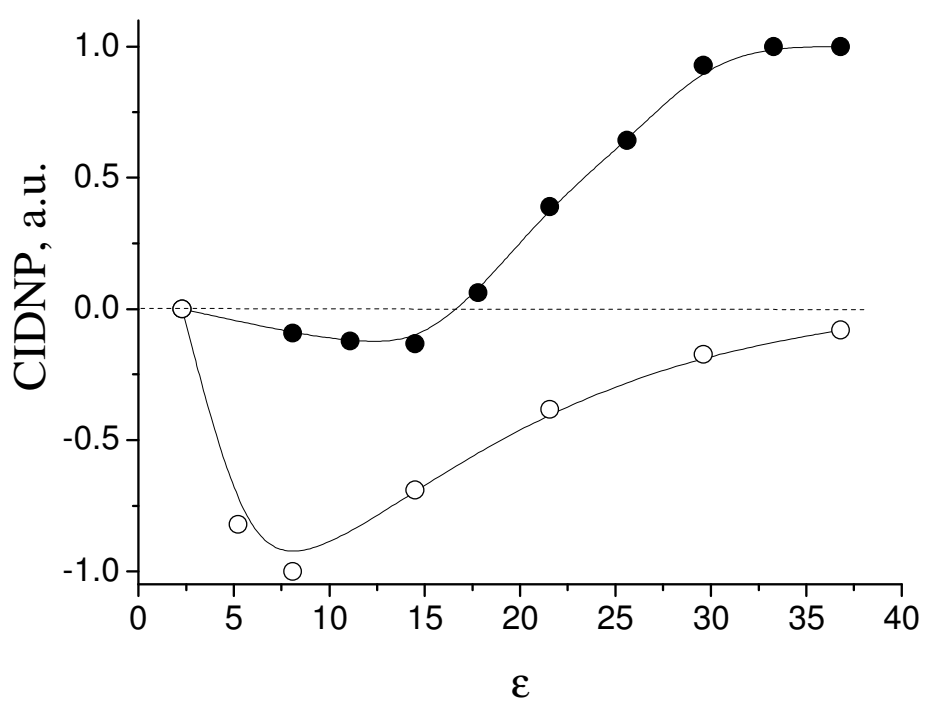

Figure 10. Dependencies of CIDNP for the pyrrolidine fragment on solvent polarity: a) for $(R, S)$-NPX-Pyr from Figure $6 a(0)$, and b) Me-NPX + Ac-Pyr system from Figure $8 b$ $(\bullet)$.

Taking into account the significance of latter conclusion, we made an attempt to consider theoretically possible reasons of the difference between the profiles of curves from figure 10. As mentioned above, all energetic characteristics and magneto - resonance parameters of the systems from fig.10 are identical. Consequently one can expect the same set of recombination rate constants for these systems. Then the main difference between quenching processes that occur in the dyad and in the encountering partners in the solution, might be connected with peculiarities of molecular dynamics: the motion of the dyad's partners relative to each other.

Spin-dependent recombination probability for a radical ion pair where both singlet and triplet states are reactive was considered earlier using Green function theory [35]. In the 
framework of this consideration, an equation for the sign of CIDNP produced by both $\mathrm{S}$ and $\mathrm{T}$ recombination channels of the RIP can be obtained from $[36,37]$. The sign is determined as:

$$
\operatorname{sign} \approx \mathrm{k}_{\mathrm{S}}-\mathrm{k}_{\mathrm{T}}+2 \tau_{\mathrm{p}} \mathrm{k}_{\mathrm{S}} \mathrm{k}_{\mathrm{T}}-\frac{\mathrm{k}_{\mathrm{S}}+\mathrm{k}_{\mathrm{T}}+2 \tau_{\mathrm{p}} \mathrm{k}_{\mathrm{S}} \mathrm{k}_{\mathrm{T}}}{\left(1+\mathrm{k}_{\mathrm{S}} \tau_{\mathrm{p}}\right)\left(1+\mathrm{k}_{\mathrm{T}} \tau_{\mathrm{p}}\right)}
$$

Here $\mathrm{k}_{\mathrm{S}}$ and $\mathrm{k}_{\mathrm{T}}$ are recombination rate constants from singlet and triplet states of the RIP and $\tau_{\mathrm{p}}$ is time the radicals dwell in the reaction zone. Due to the same recombination rate constant set the main varying parameter in this consideration is $\tau_{\mathrm{p}}$. Because the link between the donor and acceptor in the dyad undoubtedly limits the degree of freedom, the values of $\tau_{\mathrm{p}}$ in the biradical ion and RIP might really be different.

The change of CIDNP sign with solvent polarity shown in fig 10 suggests that the magnitudes of $\mathrm{k}_{\mathrm{S}}$ and $\mathrm{k}_{\mathrm{T}}$ will be approximately equal in both systems. The assumption that $\mathrm{k}_{\mathrm{S}} \square \mathrm{k}_{\mathrm{T}}$ nothing can be read between $\mathrm{kS}$ and $\mathrm{kT}$ leads to the following approximation:

$$
\operatorname{sign} \approx 2 \mathrm{k}_{\mathrm{S}}\left(\frac{1}{1+\mathrm{k}_{\mathrm{S}} \tau_{\mathrm{p}}}-\mathrm{k}_{\mathrm{S}} \tau_{\mathrm{p}}\right)
$$

As is obvious from this approximation the change of lifetime can indeed change the sign of CIDNP. This result means that in the system under study, the NPX-Pyr dyad, the long $\tau_{\mathrm{p}}$ of the biradical ion in comparison with the RIP will lead to small contribution from the triplet channel in the observed CIDNP at high solvent polarity. Then CIDNP of the dyad would correspond to the case $\lambda_{S}>\lambda_{T}$ in the entire range of polarity as observed in experiment.

Taking into account the absence of any significant external magnetic field influence on triplet yield in the NPX-Pyr dyad we can suggest that the main reason for this is that one channel of triplet formation is internal intersystem conversion of exciplex ( $\left.\mathrm{k}_{\text {exp-isc }}\right)$. The process of the dyad triplet state formation via exciplex is shown in scheme 2 and the energy diagram (Figure 9). According to scheme 2 the absence of MFE will result from the compensation of the effects with opposite signs arising from the recombination of $\mathrm{T}$ and $\mathrm{S}$ states of the biradical ion. Note that examples of small MFE's detected in systems that involve exciplex, in which high internal intersystem conversion takes place, have been described in literature. Thus, reference data is available about molecular dynamics and MFE's detected by T-T absorption or luminescence in the processes of quenching of the excited states of aromatic hydrocarbons by aromatic amines [38-41]. The analysis showed that the range of MFE's in such systems first of all is determined by the efficiency of the internal S-T conversion in exciplex from which RIP were produced. Indeed, there are very weak MFE's (2-4\%) on T-T absorption of anthracene detected by 
quenching of its excited state by dimethylaniline. At the same time high internal intersystem conversion in "anthracene-dimethylaniline" exciplex was established by Mataga [41].

Thus the investigation of the elementary mechanism of quenching of chromophore excited state in the NPX-Pyr dyad and in related systems by means of spin chemistry methods points to following peculiarities detected in the linked system. First of all it is the involvement of singlet exciplex of the dyad along with the intramolecular PET in quenching processes. Another characteristic feature is connected with long lifetime of the biradical ion in comparison with lifetime of RIP formed by the diffusion quenching processes.

As for the nature of the abovementioned influence of chiral centers on chemical reactivity of NPX-Pyr dyads, spin chemistry study allows us to make some suggestions. At first we were under the impression that the most plausible reason of the difference between the rates of quenching of the excitation of the chomophore of the $(R, S)$ - and $(S, S)$-NPX-Pyr dyads could be the difference of the energy of electron exchange interaction in the biradical ions of $(R, S)$ - and $(S, S)$ - enantiomers. In this case the stereoselectivity might be the result of different rates of back electron transfer in biradical ions of the isomers. The way of the possible influence of back electron transfer rates on fluorescence quenching, connected with existence of equilibrium between the exciplex and the biradical ion, is shown in scheme 2. However, the small intensity of CIDNP for the dyads detected in solvents with high polarity, the identity of the CIDNP effects for the stereoisomers, and the absence of the influence of external MF on triplet yield of the dyads make us conclude that processes in the biradical ions are not the main source of the stereo selectivity. Then it might be reasonable to consider the possibility of processes in the exciplex of the dyads to influence the rate of fluorescence quenching. The abovementioned internal singlet triplet conversion of exciplex has to be highly sensitive to both energetic and steric effects [42].

It is believed that future investigations of the source of the influence of chiral centers on photochemistry of linked systems have to involve the study of processes both in the biradical ion and in the exciplex .

\section{Acknowledgment}

This work was supported by the grants 08-03-00372 and 11-03-01104 of Russian Foundation of Basic Research, and the grant of Priority Programs of RAS, No 5.1.5. 


\section{Figure captions}

Figure 1. Calculated changes in the heat of formation and the distance between the donor and acceptor groups of a) (R,S)-NPX-Pyr and b) (S,S)-NPX-Pyr as a function of the torsion angle $(\phi)$ of $\mathrm{CH}_{3}-\mathrm{C}-\mathrm{C}=\mathrm{O}$ bond.

Figure 2. Kinetics of transient absorption of (R,S)-NPX-Pyr triplet state detected after laser pulse $\left(\lambda_{\mathrm{obs}}=440 \mathrm{~nm}\right)$. Inset shows the initial part of the kinetics. Arrow indicates time delay for monitoring of MFE.

Figure 3. Magnetic field dependence of the yield of (R,S)-NPX-Pyr triplet state in acetonitrile measured by laser flash photolysis.

Figure 4. Dependencies of the free energy of NPX-Pyr biradical ion (a) and exciplex (b) on dielectric constant of solvent $(\varepsilon)$. Dashed line shows the energy of excited singlet state of NPX-Pyr.

Figure 5. NMR (top) and TR CIDNP at $1 \mu$ s delay (bottom) spectra detected after laser irradiation of $(\mathrm{S}, \mathrm{S})-\mathrm{NPX}-\mathrm{Pyr}(1 \mathrm{mM})$ in $\mathrm{CD}_{3} \mathrm{CN}$.

Figure 6. Dependencies of the TR CIDNP (delay 1us) intensity for $\mathrm{N}-\mathrm{CH}_{3}$ protons of NPX-Pyr dyad (1 mM) on solvent polarity in the absence (a, left: ( - )$(\mathrm{R}, \mathrm{S})-$ and (O)- (S,S)-dyad) and in the presence of TEA (10mM) (b, right: (०)- (R,S)-dyad and ( $\mathbf{-})-\mathrm{N}-\mathrm{CH}_{2}$ protons of TEA).

Figure 7. Dependence of the TR CIDNP (delay 1us) intensity for $\mathrm{N}-\mathrm{CH}_{2}$ protons of TEA on solvent polarity in its photoreaction with DMN.

Figure 8. Dependencies of the TR CIDNP (delay 1us) intensity on solvent polarity in the systems: a) Me-NPX and TEA ( $)$; b) Me-NPX and Ac-Pyr (•).

Figure 9. Energy diagrams for the photoinduced processes in the NPX-Pyr dyad in the presence of TEA: (left) - low polarity, (right) - high polarity.

Figure 10. Dependencies of CIDNP for the pyrrolidine fragment on solvent polarity: a) for (R,S)-NPX-Pyr from Figure 6a (o), and b) Me-NPX + AcPyr system from Figure $8 b(\bullet)$. 


\section{Schemes legends}

Scheme 1. Intramolecular electron transfer in NPX-Pyr dyad.

Scheme 2. Quenching of the excited singlet state of the NPX-Pyr dyad in solution.

Scheme 3. Quenching of the excited singlet state of the NPX-Pyr dyad in solution in the presence of TEA. 


\section{References}

[1] Park, J.S., Karnas, E., Ohkubo, K., Chen, P., Kadish, K.M., Fukuzumi, S., Bielawski, C.W., Hudnall, T.W., Lynch, V.M., Sessler, J.L., Science, 329 1324-27 (2010)

[2] Reece, S.Y., Nocera, D.G., Annual Review of Biochemistry, 78, 673-99 (2009)

[3] Afanasyeva, M.S., Taraban, M.B., Purtov, P.A., Leshina, T.V., Grissom, C.B., J. Am. Chem. Soc., 128, 8651-58 (2006)

[4] Fox, M.A., Chanon, M., Photoinduced electron transfer. C: Photoinduced electron transfer reactions: organic substrates, (Elsevier, New York,1988), pp. 754

[5] Hayball, P.J., Nation, R.L., Bochner, F., Chirality, 4, 484-87 (1992)

[6] Suesa, N., Fernandez, M.F., Gutierrez, M., Rufat, M.J., Rotllan, E., Calvo, L., Mauleon, D., Carganico, G., Chirality, 5, 589-95 (1993)

[7] Evans, A.M., J. Clin. Pharmacol., 36, 7-15 (1996)

[8] Inoue, Y., Wada, T., Asaoka, S., Sato, H., Pete, J.-P., Chem. Commun. , 4, 251-59 (2000)

[9] Yorozu, T., Hayashi, K., Irie, M., J. Am. Chem. Soc., 103, 5480-548 (1981)

[10] Turro, N.J., Modern Molecular Photochemistry, (Benjamin/Cummings, 1978), pp. 628

[11] Salikhov, K.M., Molin, Y.N., Sagdeev, R.Z., Buchachenko, A.L., Spin Polarization and Magnetic Field Effects in Radical Reactions, (Akademiai Kiado, Budapest,1984), pp. 419

[12] Weiss, E.A., Ratner, M.A., Wasielewski, M.R., J. Phys. Chem. A., 107, 3639-47 (2003)

[13] Lukas, A.S., Bushard, P.J., Weiss, E.A., Wasielewski, M.R., J. Am. Chem. Soc. , 125, 3921-30 (2003)

[14] Nakagaki, R., Mutai, K., Hiramatsu, M., Tukada, H., Nakakura, S., Canadian Journal of Chemistry, 66, 1989-96 (1988)

[15] Jim'enez, M.C., Pischel, U., Miranda, M.A., J. Photochem. Photobiol. C: Photochemistry Reviews, 8, 128-42 (2007)

[16] Abad, S., Pischel, U., Miranda, M.A., Photochem. Photobiol. Sci. , 4, 69-74 (2005)

[17] Pischel, U., Abad, S., Domingo, L.R., Bosca, F., Miranda, M.A., Angew. Chem., Int. Ed.,, 42, 2531-34 (2003)

[18] Closs, G.L., Miller, R.J., J. Am. Chem. Soc., 101, 1639-41 (1979)

[19] Closs, G.L., Miller, R.J., J. Am. Chem. Soc., 103, 3586-88 (1981)

[20] Goez, M., Chem. Phys. Lett., 188, 451-56 (1992)

[21] Molokov, I.F., Tsentalovich, Y.P., Yurkovskaya, A.V., Sagdeev, R.Z., J. Photochem. Photobiol., A, 110, 159-65 (1997)

[22] Pischel, U., Abad, S., Miranda, M.A., Chem. Commun., 9, 1088-89 (2003)

[23] Hayashi, H., Nagakura, S., Bulletin of the Chemical Society of Japan 57, 322-28 (1984) 
[24] Sakaguchi, Y., Hayashi, H., Nagakura, S., Bulletin of the Chemical Society of Japan, 53, 39-42 (1980)

[25] Yonemura, H., Nakamura, H., Matsuo, T., Chemical Physics Letters, 155, 157-61 (1989)

[26] Hata, N., Hokawa, M., Chemistry Letters, 10 507-10 (1981)

[27] Magin, I.M., Kruppa, A.I., Purtov, P.A., Chemical Physics Letters, 365, 8084 (2009)

[28] Mann, C.K., Barnes, K.K., Electrochemical reactions in nonaqueous systems, (M. Dekker, New York,1970), pp. 560

[29] Bargon, J., J. Am. Chem. Soc., 99, 8350-51 (1977)

[30] Goez, M., Frisch, I., J. Phys. Chem. A. , 106, 8079-84 (2002)

[31] Chibisov, A.K., Russ. Chem. Rev., 50 615-29 (1981)

[32] Goodman, J., Peters, K., J. Am. Chem. Soc., 107, 1441 -42 (1985)

[33] Cao, H., Fujiwara, Y., Haino, T., Fukazawa, Y., Tung, C.-H., Tanimoto, Y., Bull. Chem. Soc. Japan, 69, 2801-13 (1996)

[34] Kruppa, A.I., Mikhailovskaya, O.I., Leshina, T.V., Chemical Physics Letters, 147, 65-71 (1988)

[35] Purtov, P.A., Doktorov, A.B., Chem. Phys., 178, 47-65 (1993)

[36] Doktorov, A.B., Mikhailov, S.A., Purtov, P.A., Chem. Phys., 160, 223-37 (1992)

[37] Magin, I.M., Purtov, P.A., Kruppa, A.I., Leshina, T.V., J. Phys. Chem. A., 109, 7396-401 (2005)

[38] Michel-Beyerle, M.E., Haberkorn, R., Bube, W., Steffens, E., Schröder, H., Neusser, H.J., Schlag, E.W., Seidlitz, H., Chemical Physics, 17, 139-45 (1976)

[39] Schulten, K., Staerk, H., Weller, A., Werner, H.-J., Nickel, B., Z. Physic Chem., 101, 371-90 (1976)

[40] Gnadig, K., Eisenthal, K.B., Chemical Physics Letters, 46, 339-42 (1977)

[41] Nishimura, T., Nakashima, N., Mataga, N., Chemical Physics Letters, 46, 334-38 (1977)

[42] Kuzmin, M. G., Soboleva, I. V., Dolotova, E. V., Dogadkin, D. N., High Energy Chemistry, 39, 86-96 (2005). 